
the elbow (UME) are poorly defined. We compared 112 electrodiagnostic reports which met criteria for definite or probable UME to 104 reports which excluded UME. Male gender was strongly associated with definite $(\mathrm{OR}=6.9$, $95 \% \mathrm{Cl}=2.4-20.4 ; P<0.001)$ and all UME $(\mathrm{OR}=2.2,95 \% \mathrm{Cl}=1.2-4.1 ; P$ $=0.010)$ after controlling for age and body mass index (BMI). Among men, UME was associated with increasing age $(P=0.008)$ but not a decreased BMI. Women, however, demonstrated an association between decreased $\mathrm{BMI}$ and $\mathrm{UME}(\mathrm{OR}=2.3,95 \% \mathrm{Cl}=1.3-4.2$ for $\mathrm{BMI} \leq 22.0$ versus $>22.0)$. These findings, in conjunction with gender differences in ulnar motor nerve parameters among UME subjects and controls, suggest that the pathophysiology of UME differs with gender.

(C) 2001 John Wiley \& Sons, Inc. Muscle Nerve 24: 551-554, 2001

\title{
GENDER, BODY MASS AND AGE AS RISK FACTORS FOR ULNAR MONONEUROPATHY AT THE ELBOW
}

\author{
JAMES K. RICHARDSON, MD, DONALD F. GREEN, MD, \\ SARAH C. JAMIESON, MD, and F. CLIFFORD VALENTIN, MD \\ University of Michigan Medical Center, Department of Physical Medicine and \\ Rehabilitation, MPB D5200, Ann Arbor, Michigan 48109-0718, USA
}

Accepted 23 October 2000

The pathophysiology of ulnar mononeuropathy at the elbow (UME) is likely heterogenous, with several different potential locations for entrapment. ${ }^{7}$ Despite various known predisposing factors, many patients develop UME with no obvious inciting factor or anatomical predisposition. ${ }^{14}$ In these cases of UME, which are labeled "idiopathic," the location of ulnar entrapment is thought to be at the epicondylar groove or the cubital tunnel. ${ }^{14,15}$ Such patients are regarded as "susceptible" ${ }^{15}$ to nerve injury, but for uncertain reasons.

The purpose of the present study was to compare characteristics of patients with and without UME in an effort to gain insight into predisposing traits. We reasoned that thin persons would have less mechanical protection of the ulnar nerve and would therefore sustain increased trauma to the nerve for a given amount of activity (flexion/extension or compression) as compared to heavier persons. We also reasoned that older persons repair peripheral nerve

Abbreviations: BMI, body mass index; CMAP, compound muscle action potential; UME, ulnar mononeuropathy at the elbow

Key words: body mass index; gender; mononeuropathy; risk factors; ulnar

Correspondence to: J.K. Richardson; e-mail: jkrich@umich.edu

(c) 2001 John Wiley \& Sons, Inc. tissue more slowly, thus predisposing them to UME. Other work has identified associations between body mass index, age, and gender with median mononeuropathy at the wrist (carpal tunnel syndrome). ${ }^{13,17}$ We therefore hypothesized that patients with UME would demonstrate a decreased body mass index (BMI) and an increased age compared to a control group of patients. To investigate this, we identified reports of approximately 100 patients with electrodiagnostically confirmed UME and compared them with data from a similarly sized group of patients with normal electrodiagnostic studies.

\section{METHODS}

Case Selection. Electrodiagnostic case reports of UME and controls were obtained from an electronic database of electrodiagnostic study results performed at the University of Michigan since 1988. The studies are coded by diagnoses. The reports include the following information which was used for analysis: a focused history and physical examination; patient age, gender, height, and weight; and nerve conduction parameters and results of needle electromyography. All patients were referred for electrodiagnostic evaluation of upper extremity pain or numbness.

Inclusion criteria for reports were the presence 
of a needle electromyographic examination, a median sensory response, and ulnar motor conduction studies of the forearm (below elbow) and across the elbow. Exclusion criteria for both groups included other mononeuropathy, diabetes mellitus, connective tissue disease, upper extremity trauma or fracture, cervical radiculopathy or plexopathy, inpatient status, hemiparesis or paraparesis, and use of an assistive device. Approximately 25 cases of UME were obtained from each year from 1996-1999; controls were taken from the same time periods. The control subjects consisted of approximately the first 25 subjects who satisfied inclusion criteria in each year of the study.

Studies were defined as UME cases if they were coded as UME and met two or three of the following criteria $^{5}$ : (1) ulnar conduction velocity across the elbow $<50 \mathrm{~m} / \mathrm{s}$; (2) ulnar conduction velocity across the elbow decreased by $>10 \mathrm{~m} / \mathrm{s}$ compared to the forearm (with the elbow flexed to $90^{\circ}$ ); and (3) a decrease in compound muscle action potential (CMAP) amplitude of $>20 \%$ when stimulating the ulnar nerve above compared to below the elbow.

Cases of UME were considered "definite" if all three criteria were met, and "probable" if two of three criteria were met. Controls demonstrated completely normal ulnar nerve conduction parameters across the elbow; i.e., none of the three criteria were present.

Statistical Analysis. Study subjects were divided into three diagnostic groups-definite UME, probable UME, and control. Analysis of variance was used to determine diagnostic group by BMI and diagnostic group by age interactions. The "definite" and "probable" UME diagnostic groups were then combined, and age and BMI compared with the control group (Student's $t$-test). BMI was also analyzed at intervals similar to those used by Werner et al., ${ }^{17}$ comparing subjects with $\mathrm{BMI} \leq 22.0$, between 22.0 and 29.0 , and $\geq 29.0$ using chi-square analysis to determine differences in frequency of UME among the BMI groups. Correlation coefficients between diagnostic groups and specific electrodiagnostic parameters were determined using Pearson coefficients.

Logistic regression analysis was performed using the presence or absence of definite UME, and definite and probable UME, as dependent variables. Independent variables analyzed included BMI, gender, and age. Similarly, multiple regression analysis was used to confirm correlations between diagnostic groups and electrodiagnostic parameters. The dependent variable was the electrodiagnostic measure in question and the independent variables were gender, height, and age.

A $P$ value of $<0.05$ was considered significant, whereas a value of $\geq 0.05$ and $<0.10$ was considered a trend.

\section{RESULTS}

A total of 216 subjects (115 women) were studied; 112 were UME cases (25 definite UME and 87 probable UME) and 104 were controls. The mean $( \pm$ SD) age and BMI of the subjects were $45.2( \pm 14.0)$ years and $26.8( \pm 5.5)$, respectively. When all subjects were considered together, there were no significant associations between BMI and diagnostic group [definite UME, 27.5 ( \pm 5.2$)$; probable UME, 26.7 ( \pm 6.2$)$; control, $26.7( \pm 5.0) ; P=0.745]$, or between age and diagnostic group [definite UME, 43.6 ( \pm 12.7); probable UME, $47.2( \pm 14.7)$; control, 43.8 ( \pm 13.7$) ; P=$ $0.216]$.

There were significantly greater numbers of men than women in the UME groups compared to the control group $(P<0.001$; Table 1$)$. Therefore separate analyses for men and women were performed. Men with definite and probable UME were significantly older than the controls $[48.8( \pm 13.3)$ vs. 41.8 $( \pm 14.0)$ years, respectively; $P=0.015]$. This was not true for the women $[42.9( \pm 15.1)$ vs. $44.8( \pm 13.5)$ years; $P=0.49]$. Furthermore, among men with UME there were significant negative correlations between BMI and ulnar amplitudes above $(-0.242, P=0.019)$ and below $(-0.225, P=0.013)$ the elbow, and ulnar conduction velocities in the forearm $(-0.231, P=$ 0.025 ) when controlling for age. None of these correlations was found among the women subjects with UME $(0.011, P=0.903 ; 0.004, P=0.964 ; 0.088, P=$ 0.349 , respectively).

Women with $\mathrm{BMI} \leq 22.0$ were significantly more likely to have definite or probable UME compared to the women with BMI > 22.0. This was true when the heavier women were considered as one group all with $\mathrm{BMI} \geq 22.0[P=0.004 ;$ OR $(95 \% \mathrm{CI})=3.3$ $(1.4-7.7)]$ or divided into two groups $(P=0.008)$ (Table 2). There was no difference in frequency of

\begin{tabular}{lccccc}
\hline \multicolumn{5}{c}{ Table 1. Diagnostic group by gender. } & \\
\hline & UME & UME & & & \\
Definite & Probable & All UME & Control & \\
& $(\%)$ & $(\%)$ & $(\%)$ & $(\%)$ & P value \\
\hline Women & $6(5.2)$ & $40(34.8)$ & $46(40)$ & $69(60.0)$ & \\
Men & $19(18.8)$ & $47(46.5)$ & $66(65)$ & $35(34.7)$ & $<0.001^{*}$ \\
\hline
\end{tabular}

UME, ulnar mononeuropathy at the elbow. (The definition of the different diagnostic categories is described in the text.)

${ }^{*}$ Chi-square analysis, men more likely to have UME than women. 


\begin{tabular}{|c|c|c|c|c|}
\hline & $\leq 22.0(\%)$ & $\begin{array}{l}>22.0 \text { and } \\
<29.0(\%)\end{array}$ & $\geq 29.0(\%)$ & $\begin{array}{c}P \text { value; OR, } \\
(95 \% \mathrm{Cl})\end{array}$ \\
\hline \multicolumn{5}{|l|}{ Women } \\
\hline UME & $20(60.6)$ & $14(26.9)$ & $12(40)$ & \multirow{2}{*}{$\begin{array}{r}0.004 ; 2.3 \\
(1.3-4.2)^{*,+}\end{array}$} \\
\hline Control & $13(40.4)$ & $38(73.1)$ & $18(60)$ & \\
\hline \multicolumn{5}{|l|}{ Men } \\
\hline UME & $6(54.5)$ & $36(61)$ & $23(76.7)$ & \multirow{2}{*}{$\begin{array}{r}0.109 ; 1.8 \\
(0.85-3.7)\end{array}$} \\
\hline Control & $5(44.5)$ & 23 (39) & 7 (23.3) & \\
\hline
\end{tabular}

BMI, body mass index; UME, ulnar mononeuropathy at the elbow. (The definite and probable diagnostic categories of UME have been combined.)

${ }^{*}$ Chi square analysis, women with $B M I \leq 22.0$ significantly more likely to have UME than women with $\mathrm{BMI}>22.0$.

${ }^{+}$When women with BMI between 22.0 and 29.0 and $B M I \geq 29.0$ maintained in separate groups, women with $B M I<22.0$ still more likely to have UME (chi-square analysis, $P=0.008$ )

UME based on BMI grouping among men, or for the entire group.

Logistic regression using age, gender, and BMI as independent variables demonstrated that male gender significantly increased the probability of having definite UME $(\mathrm{OR}=6.9,95 \% \mathrm{CI}=2.4-20.4 ; P<$ $0.001)$, and definite or probable UME $(\mathrm{OR}=2.2$, $95 \%$ CI $=1.2-4.1 ; P=0.010)$. In addition, logistic regression identified an age effect for men, but not women, with increasing age significantly increasing the probability of having UME $(P=0.008)$.

The control subjects' nerve conduction parameters were analyzed in an effort to detect a subclinical gender effect. On univariate analysis, women demonstrated an increased conduction velocity below the elbow as compared to men $[62.0( \pm 5.2) \mathrm{m} / \mathrm{s}$ vs. 59.5 ( \pm 4.7$) ; P=0.019]$. Using multiple regression analysis to control for height and age, ulnar motor conduction velocity below the elbow remained significantly greater among women than men control subjects $(P=0.002)$.

\section{DISCUSSION}

The major finding of this study was that men were more likely to have an UME than women. To our knowledge, this has not been reported previously. Consistent with one of our hypotheses, men with UME were significantly older than men with normal nerve conduction studies. This finding is not surprising given that age is also a risk factor for carpal tunnel syndrome, ${ }^{13,17}$ and that there is a well-documented age-associated decrement in peripheral nerve function. ${ }^{3}$

Significantly more women with a BMI $\leq 22.0$ had UME compared to women with a BMI $>22.0$, suggesting that thin women are at increased risk for UME. However, UME was not associated with a decreased
BMI among men despite the belief that compression of the elbow against a hard surface is an important cause of UME. ${ }^{1,12}$ Taken together, the data suggest that external compression at the elbow is a more important cause of UME among women than men.

Other findings suggest a gender difference with regard to UME and the ulnar nerve. Among the men with UME, ulnar conduction velocity in the forearm, and CMAP amplitudes when stimulating above as well as below the elbow, correlated significantly and inversely with BMI. None of these inverse correlations was demonstrated for the women, suggesting that technical influences related to testing obese patients is not an explanation. Furthermore, when controlling for height and age, the women control subjects had an increased ulnar conduction velocity in the forearm compared to the men.

It is commonly accepted that the two most common sites for ulnar nerve entrapment are the condylar groove and cubital tunnel. ${ }^{14}$ Compression at the latter site occurs under the flexor carpi ulnaris aponeurosis and is markedly increased with isometric contraction of the flexor carpi ulnaris. ${ }^{16}$ After the mid-teen years, forearm muscle mass and strength are significantly greater among men than women. ${ }^{2,10}$ It is possible that men, with their thicker forearm musculature and greater grip strength, develop greater pressures over the ulnar nerve with hand usage and are therefore at increased risk for UME.

This same mechanism may explain why we did not find an association between a decreased BMI and UME among men, and why there were negative correlations between multiple ulnar nerve conduction parameters and BMI among men but not women with UME. The data suggest that, in men, any mechanical protective effect of an increased BMI for the ulnar nerve at the elbow is offset by another factor. Among a large cohort of military personnel, increased BMI correlated positively with grip strength. ${ }^{9}$ If the same is true for the population we studied, subjects with increased BMI also had an increased grip strength and greater pressure over the ulnar nerve. The importance of grip in the development of UME is further suggested by studies of string musicians which found that the left arm (the fingerboard side), which performed repetitive forceful gripping movements, was at greater risk for UME than the bow side which was subjected to repetitive elbow flexion. ${ }^{6,11}$

Other factors could influence the interpretation of our data. For example, women are known to seek medical attention more readily than men. ${ }^{8}$ Therefore, our study may overestimate the number of women with normal studies and underestimate the 
number of men with mild UME. In addition, our control group was not completely normal given that it was composed of patients with symptoms significant enough to warrant an electrodiagnostic study and, further, that the electrodiagnostician elected to perform ulnar nerve conduction studies across the elbow. Such patients may have had symptoms consistent with UME so that this study may have underestimated differences between patients with and without UME. Lastly, we could not account for occupational risk factors for UME; however, occupations known to be associated with UME are not clearly and traditionally all associated with one gender. ${ }^{4}$

J.K.R. is supported by Public Health Service grant K23 AG 0098901, and S.C.J. by Public Health Service grant T32 HD 07422-10.

\section{REFERENCES}

1. Abdel-Salam A, Eyres KS, Cleary J. Driver's elbow: a cause of ulnar neuropathy. J Hand Surg 1991;16B:436-437.

2. Barnekow-Bergkvist M, Hedberg G, Janlert U, Jansson E. Development of muscular endurance and strength from adolescence to adulthood and level of physical capacity in men and women at the age of 34 years. Scand J Med Sci Sports 1996; 6:145-155

3. Bouch P, Cattelin F, Saint-Jean O, Leger JM, Queslati S, Guez D, Moulonguet A, Brault Y, Aquino JP, Simunek P. Clinical and electrophysiological study of the peripheral nervous system in the elderly. J Neurol 1993;240:263-268.

4. Bozentka DJ. Cubital tunnel syndrome pathophysiology. Clin Orthop Rel Res 1998;351:90-94.

5. Campbell WW, Greenberg MK, Krendel DA, Pridgeon RM, Sitaram KP, Williams FH. The electrodiagnostic evaluation of patients with ulnar neuropathy at the elbow: literature review of the usefulness of nerve conduction studies and electromyography. Muscle Nerve 1999;22(suppl 8):S175-S205.

6. Charness ME. Unique upper extremity disorders of musicians. In Millender LH, Louis DS, Simmons BP, editors. Occupational disorders of the upper extremity. New York: Churchill Livingstone; 1992. p 227-252.

7. Gabel GT, Amadio PC. Reoperation for failed decompression of the ulnar nerve in the region of the elbow. J Bone Joint Surg 1990;72A:213-219.

8. Green CA, Pope CR. Gender, psychosocial factors and the use of medical services: a longitudinal analysis. Soc Sci Med 1999; 48:1363-1372.

9. Jette M, Sidney K, Lewis W. Fitness, performance and anthrompometric characteristics of 19,185 Canadian Forces personnel classified according to body mass index. Milit Med 1990;155:120-126.

10. Kanehisa H, Ikegawa S, Tsunoda N, Fukunaga T. Crosssectional areas of fat and muscle in limbs during growth and middle age. Int J Sports Med 1994;15:420-425.

11. Lederman RJ. AAEM minimonograph \#43: neuromuscular problems in the performing arts. Muscle Nerve 1994;17: 569-577.

12. Magnusson M, Pope M. Epidemiology of the neck and upper extremity. In Nordin M, Andersson GBJ, Pope M, editors. Musculoskeletal disorders in the workplace: principles and practice. Philadelphia: Mosby; 1997. p 328-335.

13. Stevens JC, Sun S, Beard CM, O'Fallon WM, Kurland LT. Carpal tunnel syndrome in Rochester, Minnesota, 1961-1980. Neurology 1988;38:134-138.

14. Stewart JB. Focal peripheral neuropathies. 3rd ed. Philadelphia: Lippincott; 2000. p 241-279.

15. Tetro AM, Pichora DR. Cubital tunnel syndrome and the painful upper extremity. Hand Clin 1996;12;665-677.

16. Werner CO, Ohlin P, Elmqvist D. Pressures recorded in ulnar neuropathy. Acta Orthop Scand 1985;56:404-406.

17. Werner RA, Albers JW, Franzblau A, Armstrong TJ. The relationship between body mass index and the diagnosis of carpal tunnel syndrome. Muscle Nerve 1994;17:632-636. 